\title{
On Gravitational Waves: Did We Simply Detect the Gravitational Effect of the Sun on the Photons Moving in the Cavity of Interferometers LIGO and VIRGO?
}

\author{
Alexandre Chaloum Elbeze \\ University Pierre et Marie Curie, Paris, France \\ Email: A77190@bezeqint.net
}

How to cite this paper: Elbeze, A.C. (2018) On Gravitational Waves: Did We Simply Detect the Gravitational Effect of the Sun on the Photons Moving in the Cavity of Interferometers LIGO and VIRGO? Journal of Modern Physics, 9, 1281-1290.

https://doi.org/10.4236/jmp.2018.96076

Received: April 10, 2018

Accepted: May 21, 2018

Published: May 24, 2018

Copyright $\odot 2018$ by author and Scientific Research Publishing Inc. This work is licensed under the Creative Commons Attribution International License (CC BY 4.0).

http://creativecommons.org/licenses/by/4.0/ (c) (i) Open Access

\begin{abstract}
On September 14, 2015 09:50:45 UTC, the two laser interferometers of the LIGO program simultaneously observed a first gravitational wave signal called GW150914. With the commissioning of the VIRGO interferometer in 2017, two other detections, GW170814 and GW170817, were observed and their positions given accurately by LIGO and VIRGO. In this article, I argue that the photons circulating in the cavities of the three interferometers of LIGO and VIRGO were sensitive to the field of attraction of the planets of our Solar System and more particularly to that of the Sun, and would not be due to a coalescence of black hole or neutron stars. The shape of the signals obtained by my interaction model (called GEAR) between the photons in the interferometer cavity and the gravitational field of the Sun is very similar to that of a compact binary coalescence, identical to those obtained by general relativity. Solving the equations of GEAR also gives the exact positions and pseudo-date of the coalescences of all the LIGO and VIRGO detections detected so far, and probably those that will come at the end of 2018 and beyond.
\end{abstract}

\section{Keywords}

Gravitational Energy Absorber Reactor, GEAR, Interferometer LIGO, Interferometer VIRGO, Law of Gravitation, Gravitational Waves, Expanding Universe Localization of Black-Hole, GEAR Model, Gravitational Waves

\section{Introduction}

The theory of general relativity shows us that a pair of black holes in orbit around each other loses energy in the form of gravitational waves (GWs). The 
two black holes approach each other, a phenomenon that can last for billions of years, before accelerating suddenly in what is called compact binary coalescence. In a fraction of a second, the two black holes then collide at a speed close to that of light and merge into a single black hole. As this one is lighter than the sum of the two initial black holes, a part of their mass (the equivalent of three Suns in the case of the detection called GW150914) is converted into GWs according to Einstein's formula $E=m c^{2}$. It is these GWs that LIGO and VIRGO may have observed. General relativity, in its gravitomagnetic form [1] [2] [3] [4] [5], allows me to show that there is a gravitational interaction between the photons present in the cavities of the interferometers and the gravitational field (here, the field of attraction) of the Sun. This alternative seems to be a more credible explanation for the signals received by LIGO and VIRGO. Indeed, the correlation in the time of the signals detected by these interferometers, the locations of the pseudo-sources of coalescences of compact binary or neutron stars, and the alignment of these events with the position of the Sun on the celestial sphere allow me to formulate the following hypothesis: Since the last commissioning of the LIGO and VIRGO interferometers in 2014, to date, it seems that no GW detection associated with the coalescence of black holes or neutron stars in terms of Einstein's general relativity has in fact been detected.

My argument is based in part on earlier work of Einstein [1], Ruggiero and Tartaglia [4], Ivashchuk and Melnikov [5], Elbeze [2] [3], Melnikov [6], De Sitter [7], and Jaupart et al. [8], and takes as a starting point results related to the framework of gravitomagnetism implied by Einstein's general relativity theory. This combines with the Newtonian radial acceleration $\gamma$ that is undergone by a test particle of mass $m$ (here, the photons present in the interferometer cavity of LIGO and VIRGO) along radius $r$ to give total acceleration $\gamma$ as follows:

$$
\gamma=\frac{G \cdot M}{r^{2}} \cdot \frac{1}{\sqrt{1-v / c}}
$$

Here, $v$ is the projection of the velocity $v$ along $r$. Note that in this study, if the gravitational constant, $G$, and $M$ (here, the mass of the Sun) are relativistic and depend on speed $v$, then $r$ does not depend on this speed and remains constant. An interesting characteristic of Equation (1) is that acceleration is no longer independent of the sign of the velocity $v$ of the test particles making up the mass $\Delta M$ of the photons in the interferometer cavity.

\section{Energy Absorbed by the Photons in LIG0's Cavities}

I argue that for all the dates of detections of GWs given by the institutions LIGO and VIRGO, the Sun was positioned according to the graph in Figure 1, that is, in the direction of the axis of the $\mathrm{X}$ arm of the LIGO Livingston Observatory (LLO), in the direction of the Y arm at LIGO Hanford Observatory (LHO), and in the direction of the $\mathrm{X}$ and $\mathrm{Y}$ arms of the VIRGO interferometer. In this article, the arms LLO (X) and LHO (Y) will be considered in parallel. 


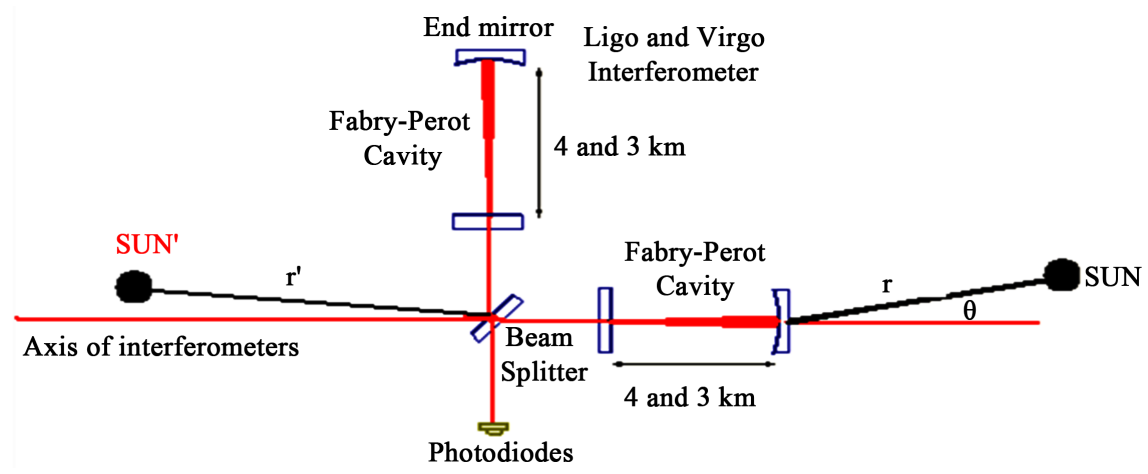

Figure 1. LIGO and VIRGO interferometers (Hanford, Livingston, and Pisa, Italy).

With GTSMHGW defined by the International Astronomical Union as the Greenwich sidereal time for the specific GW on a given day, and with $\phi s=-32.50^{\circ}$, right ascension (RA) and declination (dec.) of the Sun on the celestial sphere, the projection of the $\mathrm{x}, \mathrm{y}, \mathrm{z}$ unit vector of the Sun's position (as a function of time) at the time of detecting the GW is given by Slat $\equiv$ dec. and Slong, where Slat is the projection of the latitude in radians and Slong, given by Equation (2), is the longitude in degrees, equal to

$$
\text { Slong }=\left[-\frac{\left(G T S M H G W(t) \cdot s+\frac{\phi s}{15} \cdot 3600 s\right)}{3600 s}+R A\right] \cdot 15+180 \cdot i
$$

Here, $i$ takes the value of 1 between June 21 and December 22 and 0 for the rest of the year. This is because the calculation of Slong is shifted by $\pi$ with respect to the vernal reference point, i.e., the point of the spring equinox.

$$
\left(\begin{array}{c}
X \operatorname{sun} G W \\
Y \operatorname{sun} G W \\
Z \operatorname{sun} G W
\end{array}\right)=\left[\begin{array}{c}
\sin \left(\frac{\pi}{2}-\text { Slat }\right) \cdot \cos \left(\text { Slong } \cdot \frac{\pi}{180}\right) \\
\sin \left(\frac{\pi}{2}-\text { Slat }\right) \cdot \sin \left(\text { Slong } \cdot \frac{\pi}{180}\right) \\
\cos \left(\frac{\pi}{2}-\text { Slat }\right)
\end{array}\right]
$$

The direction of the $\mathrm{X}$ arm taken as reference for LLO and the $\mathrm{Y}$ arm for LHO is given by [9]:

$$
\left(\begin{array}{l}
X L H O Y \\
Y L H O Y \\
\text { ZLHOY }
\end{array}\right) \approx\left(\begin{array}{l}
X L L O X \\
Y L L O X \\
\text { ZLLOX }
\end{array}\right)=\left(\begin{array}{c}
-0.95457412153 \\
-0.1415807734 \\
-0.26218911324
\end{array}\right)
$$

Finally, we can calculate the $\cos \theta$ of the angle $\theta$ between the axis $r$ of the Sun and the axis of the cavities of the X arm of LLO and the Y arm of LHO at zero time of the coalescence of the GW, with wearth the rotational speed of the Earth, $t$ the time since zero time of the coalescence detection of the GW, $\phi=22.586^{\circ} \times \frac{\pi}{180}$ and $\Delta \phi \approx 2^{\circ} \times \frac{\pi}{180}$, the rotation matrix: 


$$
\left(\begin{array}{ccc}
\cos (\text { wearth } \cdot t+\phi+\Delta \phi) & -\sin (\text { wearth } \cdot t+\phi+\Delta \phi) & 0 \\
\sin (\text { wearth } \cdot t+\phi+\Delta \phi) & \cos (\text { wearth } \cdot t+\phi+\Delta \phi) & 0 \\
0 & 0 & 1
\end{array}\right)
$$

the angles $\phi$ and $\phi s$ are constants that intervene only for an exact calculation of the Sun's position, and $\Delta \phi$ is a correction angle $\phi$ dependent on the position of the Sun on the ecliptic and not exceeding the order of magnitude of $2^{\circ}$.

The rotation matrix applied to the direction vector of the X arm of LLO allows for a relatively short time to consider whether the relationship $\cos (\mathrm{XLLOGW}(t))$ (Equation (4)) is sufficiently accurate. Indeed, I consider that the position $x, y, z$ of the Sun during this time $t$ remains relatively constant, or the relationship:

$$
\begin{aligned}
& \cos (\mathrm{XLLOGW}(t)) \\
= & \left(\begin{array}{l}
X \operatorname{sun} G W \\
Y \operatorname{sun} G W \\
Z \operatorname{sun} G W
\end{array}\right) \cdot\left(\begin{array}{ccc}
\cos (\text { wearth } \cdot t+\phi+\Delta \phi) & -\sin (\text { wearth } \cdot t+\phi+\Delta \phi) & 0 \\
\sin (\text { wearth } \cdot t+\phi+\Delta \phi) & \cos (\text { wearth } \cdot t+\phi+\Delta \phi) & 0 \\
0 & 0 & 1
\end{array}\right) \\
& \cdot\left(\begin{array}{c}
X L L O X \\
Y L L O X \\
\text { ZLLOX }
\end{array}\right)
\end{aligned}
$$

Note that Equation (4) can have the sign $\pm \cos (\operatorname{XLLOGW}(t))$ because of the Sun's position (see Figure 1). Using Equation (1) and substituting the value of $\underline{v}$ by the $\cos (\mathrm{XLLOGW}(t))$ of Equation (4), the calculation of the residual energy absorbed by a photon present in the cavity of the LIGO or VIRGO interferometer remains relatively simple to calculate. Indeed, as Equation (1) depends on the sign of speed $\pm v$, the energy of the gravitational field of the Sun absorbed by the photon will depend on the number of roundtrips between the mirrors of the cavity. We can write the following:

$$
\begin{aligned}
& \cos \theta(t) \equiv \cos (\mathrm{XLLOGW}(t)) \\
& e(\text { Epx }, \text { sig }, h, t)=G \cdot \frac{M s u n}{r^{2}} \cdot \frac{E p x}{c^{2}} N r t \int_{0}^{h} \frac{\cos \theta(t)}{\sqrt{1-\operatorname{sig} \cdot \cos \theta(t)}} \\
& \cdot\left[\frac{1}{\sqrt{1-(\operatorname{sig} \cdot \cos \theta(t))^{2}}}\right]^{0.5} \cdot \mathrm{d} h
\end{aligned}
$$

In this relationship of energy absorbed during a trip $e(E p x, \operatorname{sig}, h, t), r$ represents the distance between the Sun and the cavity of the LIGO or VIRGO interferometer, Msun is the mass of the Sun, Epx is the energy of the circulating photon in the cavity (laser photon), \pm sig is the sign of speed, $\pm h$ is the distance between the two mirrors of the cavity of the interferometer, $\mathrm{Nrt}$ is the number of roundtrips, and $t$ is the time around the zero point of coalescence. The balance for a roundtrip is:

$$
\Delta E_{G W}=e(E p x, \operatorname{sig}, h, t)+e(E p x,-s i g,-h, t)
$$

The function $\Delta E_{G W}$ is similar to the energy of the GW detected by LIGO and 
VIRGO, from 2014 to 2017. With the exact time of the detection of coalescence, we can draw the graph in Figure 2.

The graph in Figure 2 shows that all the detections coincide with the alignment of the Sun on the exact dates of the coalescences of the GW with the axes of the X, Y, and XY arms of the interferometers LLO, LHO, and VIRGO, respectively.

\section{Localization of the Supposed Coalescence Sources}

To complete our demonstration of the supposed localizations (see Figure 3) of black holes and neutron stars, I maintain that the angles measured on the celestial sphere between the position of the Sun and the supposed positions of the black holes and neutron stars, as well as the angles between the black holes and neutron stars, are close to $90^{\circ}$. For this, let us write the four spherical trigonometry Equations (6)-(9) that allow the calculation of these angles and their coordinates on the celestial sphere, namely:

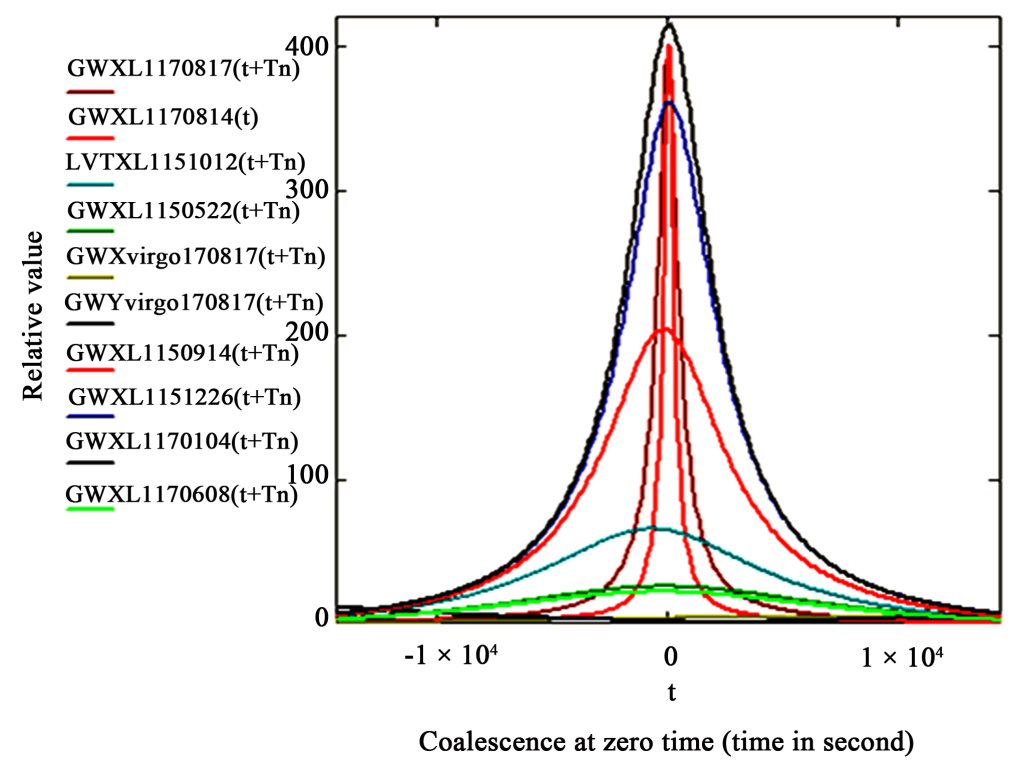

Figure 2. Graph of LIGO and VIRGO GW detections.

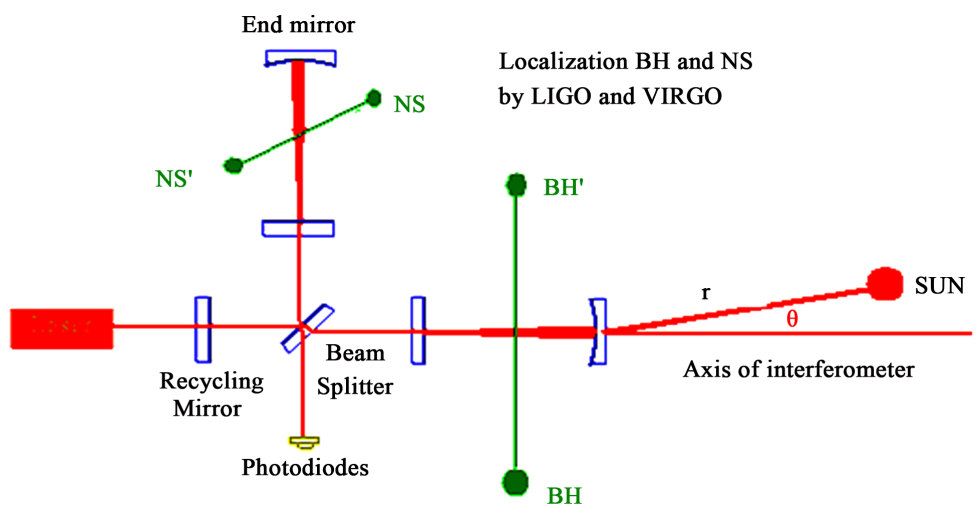

Figure 3. Localizations of GW sources by LIGO and VIRGO. 


$$
\begin{aligned}
& \cos \left(\frac{\pi}{2}-\text { Slat }\right) \cdot \cos \left(\frac{\pi}{2}-\text { BHlat }\right)+\sin \left(\frac{\pi}{2}-\text { Slat }\right) \cdot \sin \left(\frac{\pi}{2}-\text { BHlat }\right) \\
& \cdot \cos \left((\text { Slong }- \text { BHlong }) \cdot \frac{\pi}{180}\right)=0 \\
& \quad \cos \left(\frac{\pi}{2}-\text { Slat }\right) \cdot \cos \left(\frac{\pi}{2}-\text { NSlat }\right)+\sin \left(\frac{\pi}{2}-\text { Slat }\right) \cdot \sin \left(\frac{\pi}{2}-\text { NSlat }\right) \\
& \cdot \cos \left((\text { Slong }- \text { NSlong }) \cdot \frac{\pi}{180}\right)=0 \\
& \cos \left(\frac{\pi}{2}-\text { BHlat }\right) \cdot \cos \left(\frac{\pi}{2}-\text { NSlat }\right)+\sin \left(\frac{\pi}{2}-\text { BHlat }\right) \cdot \sin \left(\frac{\pi}{2}-\text { NSlat }\right) \\
& \cdot \cos (\text { BHlong }- \text { NSlong })=0 \\
& \arccos \left[\cos \left(\frac{\pi}{2}-\text { BHlat }\right)^{2}+\sin \left(\frac{\pi}{2}-\text { BHlat }\right)^{2} \cdot \cos \left[(\text { LLOlong }- \text { BHlong }) \cdot \frac{\pi}{180}\right]\right. \\
& \frac{180}{\pi} \cdot \frac{1}{\cos (\text { BHlat })}+\text { LLOlong }=\text { BHlong }
\end{aligned}
$$

In Equations (6)-(9), BHlat and NSlat represent the latitude of the black holes and neutron stars, respectively, and BHlong and NSlong are their longitude, respectively, on the celestial sphere. LLOlat and LLOlong are the coordinates of the LLO X arm on the celestial sphere, and by way of example for the detection event GW170817 (with $i=1$ ):

$$
\operatorname{astz}(t)=\left(\frac{G T S M H G W(t)}{24 \cdot 3600} \cdot 360+\phi s\right) \cdot \frac{\pi}{180}-\pi
$$

I obtained for LLOlong

$$
\begin{aligned}
\text { LLOlong }= & (\operatorname{axtz}(t)+\phi+\Delta \phi) \cdot \frac{180}{\pi} \\
& - \text { whole part of }\left(\frac{(\operatorname{axtz}(t)+\phi+\Delta \phi) \cdot \frac{180}{\pi}}{360}\right) \cdot 360 \\
& -\left(90+\frac{46}{60}+\frac{27}{3600}\right)
\end{aligned}
$$

The term $\left(90+\frac{46}{60}+\frac{27}{3600}\right)$ in Equation (10) represents the longitude of LLO [9].

For GW170817 and for $t=0 \quad$ (coalescence), I find with

LLOlat $=0.533 \mathrm{rad}$ or $30.539^{\circ}:$ LLOlong $=47.297^{\circ}$. Figure 3 shows a graph corresponding to the position of the stars in coalescence, supposing that the maximum detection is done perpendicularly along the arms of LIGO and VIRGO:

After solving Equation (9), we obtain the value of BHlat which allows us to find the value of $\mathrm{BH}$ long from Equation (6). This pair of coordinates is the point of reference, the "master" location of the black holes and neutron stars. After the 
rotation of the vector position of the master location around the axis of the Sun ( $\boldsymbol{r}$ ) (where the date coordinates are those of the GW in question) of $\frac{\pi}{2}$, then $\pi$, and finally by $\frac{3}{2} \pi$, we obtain the only four possible positions (solutions to Equations (6)-(9)) of the stars or pseudo-coalescences. Finally, we find for the detection events GW170814 and GW170817, Figure 4 and Figure 5.

\section{Calculation of the Coalescence Signal According to the GEAR Model}

The calculation, using the GEAR model, of the signal effectively received by LIGO and VIRGO is identical to a coalescence of black holes or neutron stars.

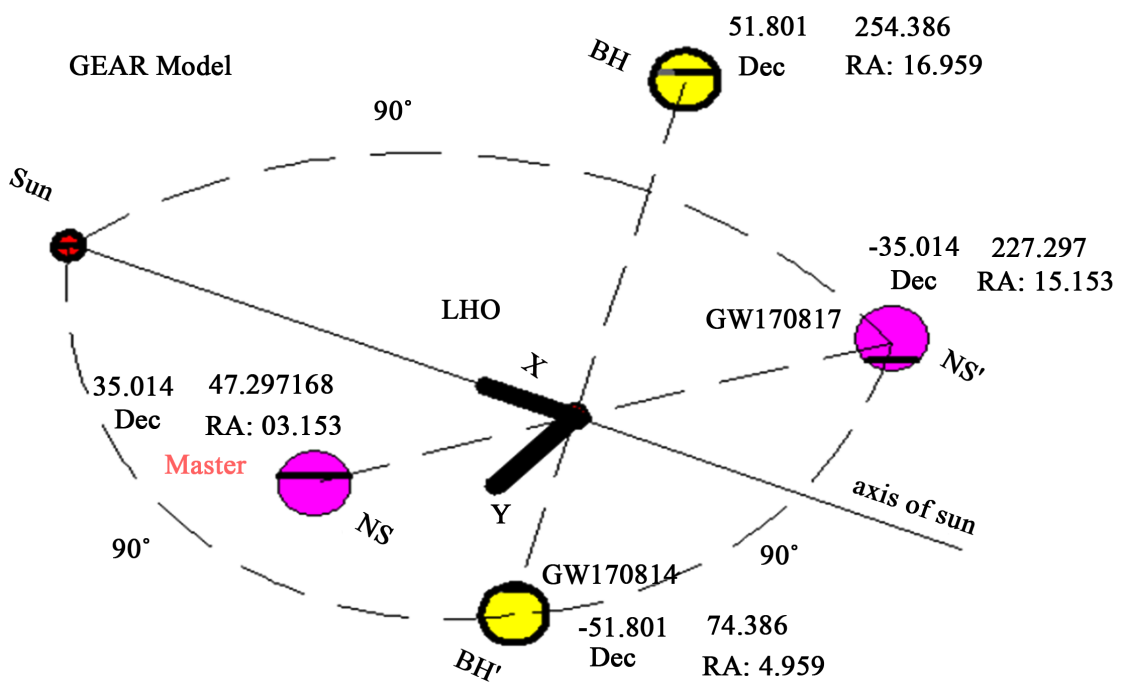

Figure 4. The values, latitudes, and longitudes of the positions of the black holes and neutron stars are the solutions of Equations (6)-(9).

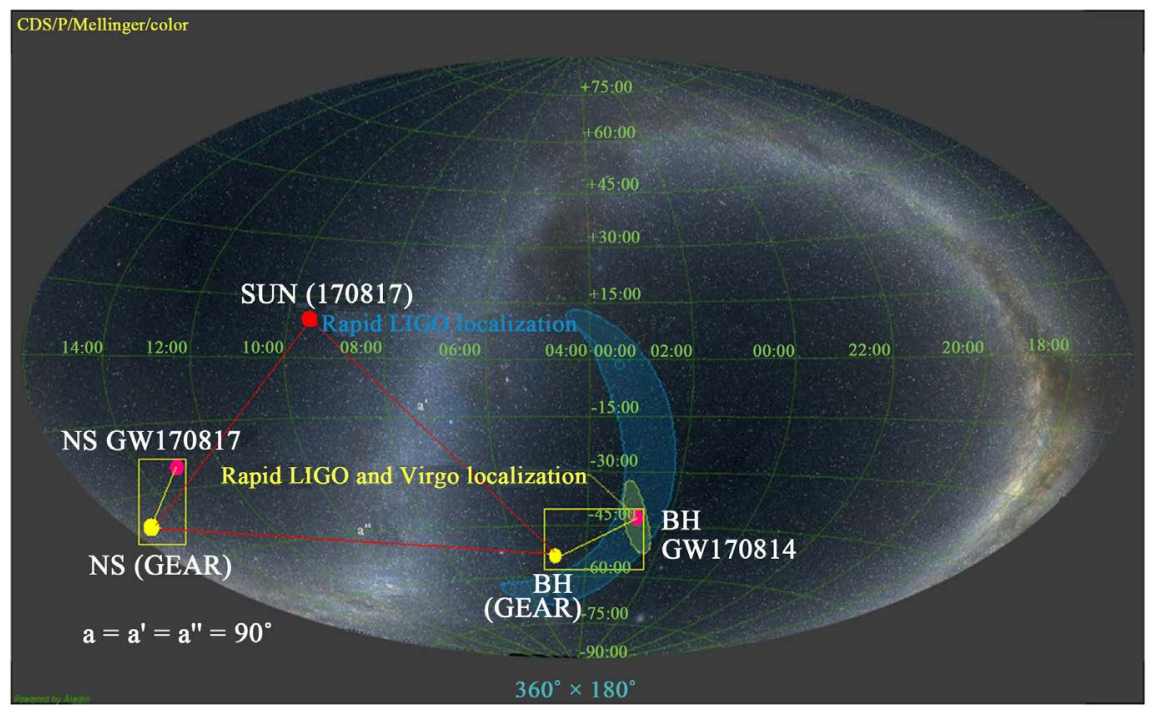

Figure 5. GEAR positions of stars coalescing on the celestial sphere, compared with data from LIGO and VIRGO. 
This signal is obtained with GEAR (Figure 6) by applying cavity quantum electrodynamics to the action of the Sun's gravitational field on the photons present in the LIGO and VIRGO cavities. The long development of the calculations will not be developed here. Figure 6 shows the signal obtained by GEAR, validated by the LIGO authorities (Rai Weiss, October 5, 2016, personal communication).

To obtain the coalescence signal of Figure 7, we must assume that the value of Equation (4) is revised upwards by a factor of 1.0005311723617234, probably due to the lack of precision of the calculations. We actually obtain for the coalescence of GW170817 the signal in Figure 7.

\section{Meaning of the Sign of $\pm \cos (\mathrm{XLLOGW}(t))$}

Figure 8 shows the combination of the direction of the Sun and the direction of the LLO arms.

In Figure 8, we see clearly that the sign of Equation (4) takes the same sign of the vector product of $\frac{\boldsymbol{r} \cdot \text { Lcavity }}{\mid \boldsymbol{r} \cdot \text { Lcavity } \mid}$, or \pm 1 , and so Equation (4) becomes $\pm \cos (\mathrm{XLLOGW}(t))$ according to the position of the Sun. We thus have the possibility of knowing the order of arrival of the signal at the interferometers of LIGO. With a negative sign for Equation (4), the signal arrives first at LLO and later at LHO; in the case of a positive sign, the signal arrives at LHO before arriving at LLO. See Table 1.

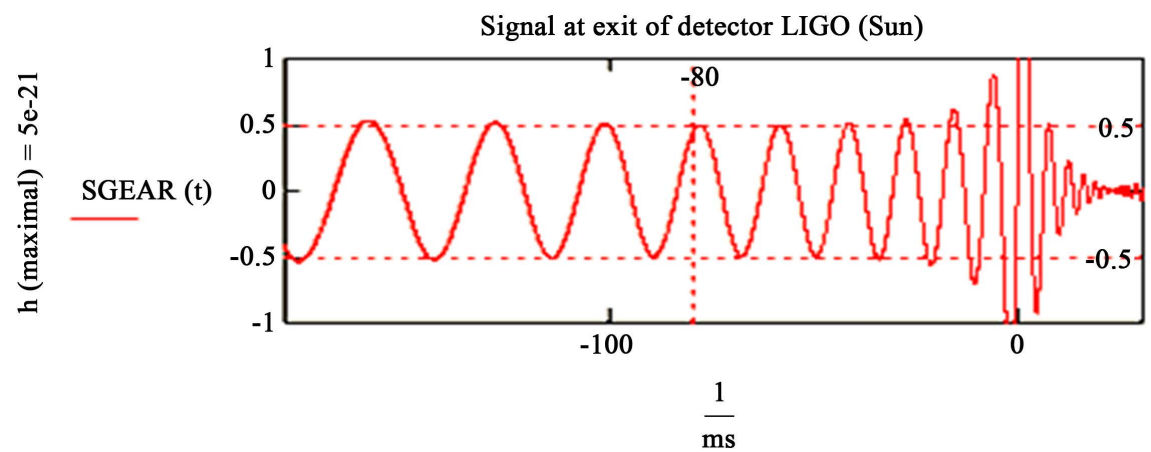

Figure 6. Black hole coalescence signal obtained by the GEAR model, the same as the signal received by LIGO and VIRGO for GW150914 (ref. [10]).

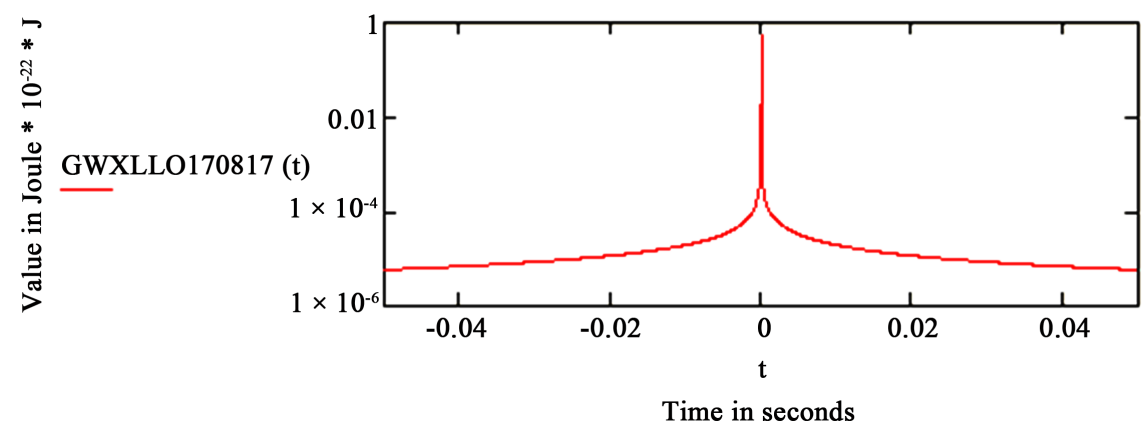

Figure 7. GW signal GW170817, obtained by GEAR. 


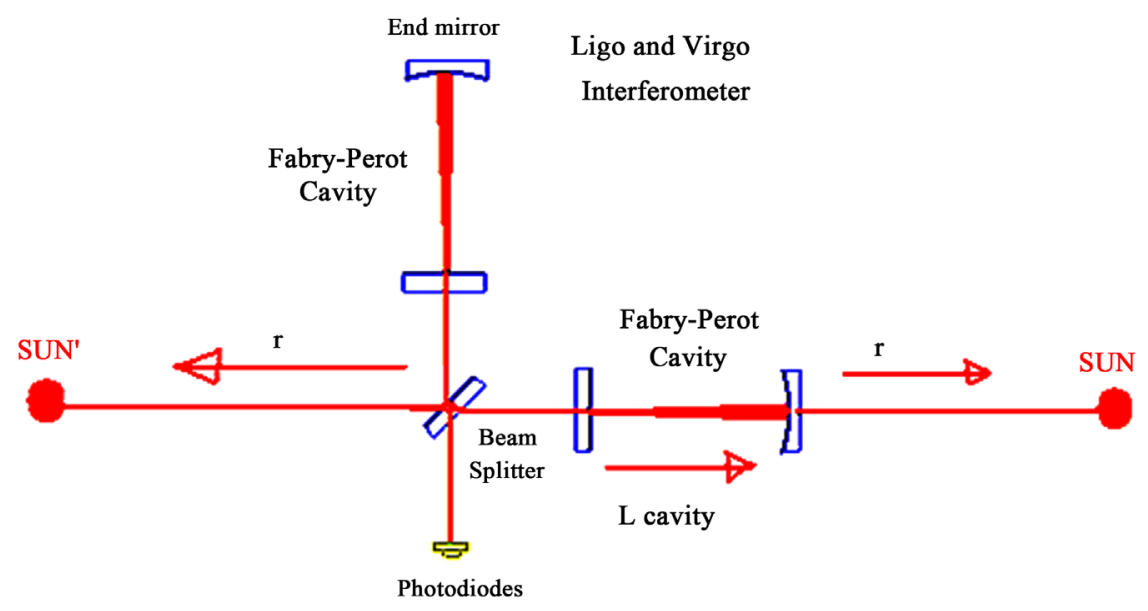

Figure 8. Direction of the Sun and the sign of $\pm \cos (\mathrm{XLLOGW}(t))$.

Table 1. Sign of Equation (4) according to the position of the sun.

\begin{tabular}{ccccc}
\hline GW & $\begin{array}{c}\text { Date } \\
(\mathrm{dd} / \mathrm{mm} / \text { yyyy })\end{array}$ & Hour UTC & $\begin{array}{c}\text { Arrival time from } \\
\text { LHO to LLO }(\mathrm{ms})\end{array}$ & $\begin{array}{c}\text { Sign of } \\
\cos (\text { XLLOGW }(t))\end{array}$ \\
\hline GW150914 & 2015 & & & - \\
LVT151012 & $14 / 09 / 2015$ & $9: 50: 45$ & -7 & - \\
GRB150522 & $22 / 10 / 2015$ & $9: 54: 43$ & & + \\
GW151002 & $02 / 10 / 2015$ & $09: 50: 45$ & & + \\
GW151226 & $26 / 12 / 2015$ & $03: 38: 53$ & +1.1 & + \\
& 2017 & $10: 23: 50$ & & + \\
GW170104 & $04 / 01 / 2017$ & $10: 11: 59$ & +3 & + \\
GW170814 & $14 / 08 / 2017$ & $10: 30: 43$ & $-14($ VIRGO) & - \\
GW170608 & $08 / 06 / 2017$ & $02: 01: 16.49$ & +7 & + \\
GW170817 & $17 / 08 / 2017$ & $12: 41: 04$ & $-0.5-?($ VIRGO) & + \\
\hline
\end{tabular}

Table 1 shows an advance signal for LLO when the sign of Equation (4) is negative, and a delay when the sign is positive. With regard to VIRGO, there remains serious doubt about the arrival of a signal of great amplitude at the same time as at LLO and LHO. The GW 170608 detection by GEAR is shifted by $\pm 12 \mathrm{~h}$ with that at LIGO, which remains to be clarified.

\section{Conclusions}

In this article, with four different reasons developed here, I have shown that it is likely that the signals detected by the LIGO and VIRGO interferometers originate from the interaction between the Sun's gravitational field and the photons present in the interferometers' cavities. In fact, LIGO and VIRGO probably did not detect signals from black hole or neutron star coalescence as defined by 
Einstein's general relativity. And Einstein may have been right in 1936 when he wrote to his friend Max Born [11]:

"Together with a young collaborator, I arrived at the interesting result that gravitational waves do not exist, though they had been assumed a certainty to the first approximation. This shows that the non-linear general relativistic field equations can tell us more or, rather, limit us more than we have believed up to now."

\section{References}

[1] Einstein, A. (1950) The Meaning of Relativity. Princeton University Press, Princeton, NJ.

[2] Elbeze, A.C. (2012) Earth, Moon, and Planets, 108, 151-163. https://doi.org/10.1007/s11038-012-9388-9

[3] Elbeze, A.C. (2013) SpringerPlus, 2, 513. https://doi.org/10.1186/2193-1801-2-513

[4] Ruggiero, M.L. and Tartaglia, A. (2002) Il Nuovo Cimento B, 117, 743.

[5] Ivashchuk, V.D. and Melnikov, V.N. (2002) Problems of G and Multidimensional Models. In: Koga, J., et al., Eds., Proceedings of JGRG11, Waseda University, Tokyo, 405-409.

[6] Melnikov, V.N. (2007) Gravitation and Cosmology, 13, 81-100.

[7] De Sitter, W. (1916) Monthly Notices of the Royal Astronomical Society, 76, 699-728. https://doi.org/10.1093/mnras/76.9.699

[8] Jaupart, C., Labrosse, S. and Mareschal, J.-C. (2007) Temperatures, Heat and Energy in the Mantle of the Earth. In: Bercovici, D., Ed., Treatise on Geophysics, Vol. 7, Mantle Convection, Elsevier, 253-303.

[9] Lazzarini, A., et al. (2001) Review of Scientific Instruments, 72, 3086-3094. https://doi.org/10.1063/1.1376138

[10] Abbott, B.P., et al. (LIGO Scientific Collaboration and Virgo Collaboration) (2016) Physical Review Letters, 116, 241102. https://doi.org/10.1103/PhysRevLett.116.241102

[11] Einstein, A. and Born, M. (2005) The Born-Einstein Letters. Friendship, Politics and Physics in Uncertain Times. Macmillan, London, UK. 